\title{
The rice white green leaf 2 gene causes defects in chloroplast development and affects the plastid ribosomal protein $\mathrm{S} 9$
}

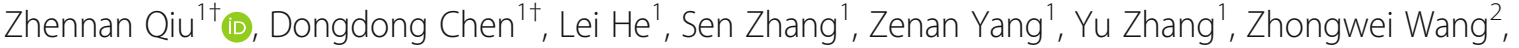 \\ Deyong Ren ${ }^{1}$, Qian Qian', Longbiao Guo ${ }^{1 *}$ and Li Zhu ${ }^{1 *}$
}

\begin{abstract}
Background: Plastid ribosomal proteins (PRPs) play important roles in the translation of key proteins involved in chloroplast development and photosynthesis. PRPs have been widely studied in many plant species; however, few studies have investigated their roles in rice.

Result: In the present study, we used ethyl methane sulfonate mutagenesis and obtained a novel rice mutant called white green leaf 2 ( $w g / 2)$. The wgl2 mutants exhibited an albino phenotype from germination through the three-leaf stage, and then gradually transitioned to green through the later developmental stages. Consistent with this albino phenotype, wg/2 mutants had abnormal chloroplasts and lower levels of photosynthetic pigments. Map-based cloning and DNA sequencing analyses of wgl2 revealed a single-nucleotide substitution (G to $T$ ) in the first exon of LOC_ Os03g55930, which resulted in a substitution of glycine 92 to valine (G92 V). WGL2 encodes a conserved ribosomal protein, which localizes to the chloroplast. Complementation and targeted deletion experiments confirmed that the point mutation in WGL2 is responsible for the wg/2 mutant phenotype. WGL2 is preferentially expressed in the leaf, and mutating WGL2 led to obvious changes in the expression of genes related to chlorophyll biosynthesis, photosynthesis, chloroplast development, and ribosome development compared with wild-type.

Conclusions: WGL2 encodes a conserved ribosomal protein, which localizes to the chloroplast. WGL2 is essential for early chloroplast development in rice. These results facilitate research that will further uncover the molecular mechanism of chloroplast development.
\end{abstract}

Keywords: Plastid ribosomal protein, Albino phenotype, Chloroplast development, Rice

\section{Background}

The development of chloroplasts from proplastids involves plastid replication and activation of plastid DNA synthesis, chloroplast genetic system "build-up", and synthesis and assembly of the photosynthetic apparatus. These steps are regulated by the coordinated expression of nuclear and plastid genes (Kusumi et al. 2010; Kusumi et al. 2011; Mullet 1993). Transcription of chloroplast genes depends on the nucleus-encoded RNA polymerase (NEP) and the plastid-encoded RNA polymerase (PEP) (He et al. 2018; Hedtke et al. 1997; Qiu et al. 2017; Shiina et al. 2005). Plastid

\footnotetext{
*Correspondence: guolongbiao@caas.cn; zhuli05@caas.cn

†Zhennan Qiu and Dongdong Chen contributed equally to this work.

'State Key Laboratory of Rice Biology, China National Rice Research Institute, Hangzhou 310006, China

Full list of author information is available at the end of the article
}

and chloroplast development are affected by NEP and PEP throughout plant growth (Qiu et al. 2017). During chloroplast development, NEP preferentially transcribes plastid housekeeping genes, such as those encoding the PEP apparatus, rRNA, and tRNA, and the overall transcriptional and translational activities in the chloroplast dramatically increase (Hajdukiewicz et al. 1997). Later in chloroplast development, NEP becomes less important and chloroplast genes are transcribed by PEP (De Santis-Maclossek et al. 1999), and translated by the ribosomal complex (Pogson and Albrecht 2011).

Ribosomes are essential for protein synthesis, and in plants the ribosomes catalyze protein synthesis in the cytoplasm, plastids, and mitochondria (Zhang et al. 2016). Plastid ribosomal proteins (PRPs) play important roles in the build-up step of chloroplast differentiation. Plastid 
proteins are translated by the $70 \mathrm{~S}$ ribosome, which consists of one small (30S) and one large (50S) ribosomal subunit; these have bacterial orthologs (Subramanian 1985). The small subunit consists of the 16S rRNA and 24 PRPs, including 12 proteins encoded by plastid genes and 12 encoded by nuclear genes (Yamaguchi and Subramanian 2003). The large subunit consists of three rRNAs (23S, 5S, and 4.5S) and 33 PRPs, including 8 proteins encoded by plastid genes and 25 encoded by nuclear genes (Yamaguchi et al. 2003). The essential and non-essential PRPs are partially conserved between bacteria and chloroplasts. Interestingly, some ribosomal proteins are not essential for the bacterial ribosome, but are essential for chloroplasts (Tiller and Bock 2014).

Since PRPs are crucial for plant growth and development, the lack of PRPs can affect protein synthesis and result in diverse phenotypes. Due to the lack of the plastid small subunit protein 17 (PRPS17), the maize high chlorophyll fluorescent 60 (hcf60) mutant has a seedling-lethal phenotype (Schultes et al. 2000). The maize lethal embryo 1 (lem1) mutant contains a mutation in the gene encoding a protein that is similar to the rice PRPS9, and lem1 mutants have an early embryonic death phenotype (Ma and Dooner 2004). The tobacco mutant plastid ribosomal protein S18 (prps18) displays defects in leaf development (Rogalski et al. 2006). The plastid-encoded proteins RPL33 and RPS15 are required under certain environmental conditions in tobacco, and improve ribosome biogenesis efficiency at elevated temperatures (Ehrnthaler et al. 2014; Rogalski et al. 2008). Plastid proteins play essential roles in embryo development, which has been shown with various plastid ribosomal protein mutants in Arabidopsis (prps5, 9, 13, 20 and prpl1, 4, 6, 10, 13, 18, 19, 21, 22, 27, 28, 31, 32, 35, 36) (Gong et al. 2013; Wang et al. 2017a). The Arabidopsis plastid ribosomal protein S5 (RPS5) plays a role in photosynthesis, plant development, and cold tolerance (Zhang et al. 2016). In rice, the PRP mutants albino seedling lethality 1 (asl1), albino seedling lethality 2 (asl2), and albino lethal 1(al1) show albino and seedling lethality phenotypes (Gong et al. 2013; Lin et al. 2015; Zhao et al. 2016). ASL1 encodes the plastid 30S ribosomal protein S20 (RPS20), ASL2 encodes the chloroplast $50 \mathrm{~S}$ ribosome protein L21, and $A L 1$ encodes the PRPL12 protein. WLP1 encodes the plastid ribosomal protein L13 and TCD11 encodes the small ribosomal subunit protein S6 (RPS6). The rice white leaf and panicles 1 (wlp1) and thermo-sensitive chlorophyll-deficient mutant 11 (tcd11) mutants display albino phenotypes at low temperatures, suggesting that both genes are required for normal chloroplast development, especially under low temperature conditions (Song et al. 2014; Wang et al. 2017a).

Although many PRPs have been studied in plants, few have been studied in rice. In the present study, we obtained a novel rice mutant, white green leaf 2 (wgl2); this mutant exhibited an albino phenotype at the seedling stage and recovered green leaves at later stages. Map-based cloning revealed that WGL2 encodes a ribosomal protein. Our results suggest that the PRP WGL2 plays a vital role in the early development of chloroplasts in rice.

\section{Results}

\section{The wg/2 mutants have an albino phenotype at the} seedling stage

A novel rice albino mutant named $w g l 2$ was isolated from the japonica rice cultivar NPB that had been subjected to ethyl methane sulfonate mutagenesis. The $w g l 2$ mutant exhibited an albino phenotype from germination through the three-leaf stage, and then gradually turned from albino to green through the later stages of development (Fig. 1a, b, c). Consistent with the albino phenotype, wgl2 mutants had lower Chl $a$, Chl $b$, and Car contents relative to the wild type (NPB). Moreover, the total chlorophyll content of $w g l 2$ and the ratio of $\mathrm{Chl}$ $a / b$ were much lower than in the wild type (Fig. 1d). When wgl2 exhibited green leaves at later stages of development, its $\mathrm{Chl}$ and Car contents recovered, but were still lower than wild type (Fig. 1e).

\section{WGL2 affects chloroplast development}

Abnormal chloroplast development usually leads to lower total chlorophyll contents (Sakamoto et al. 2009). To examine whether chloroplast development was affected in $w g l 2$ albino seedlings, we analyzed the chloroplast ultrastructure of wild-type and $w g l 2$ seedlings at the three-leaf stage with transmission electron microscopy. We observed normal chloroplasts in wild-type plants, with dense and well-structured grana stacks. By contrast, the wgl2 albino mutant had chloroplasts with disrupted architecture, as well as no stromal thylakoids or stacked grana thylakoids, instead displaying oval-shaped vesicles (Fig. 2). These results suggest that wgl2 albino mutant is severely defective in chloroplast development.

\section{Map-based cloning and sequence analysis of WGL2}

To explore the molecular mechanism responsible for the wgl2 mutant phenotype, we generated an $\mathrm{F}_{2}$ population by crossing the wgl 2 mutant with the indica cultivar TN1 to generate a segregating population. All $F_{1}$ plants displayed a normal green phenotype, and segregation occurred in the $\mathrm{F}_{2}$ plants that were selfed from the heterozygous $\mathrm{F}_{1}$ plants. Of the $528 \mathrm{~F}_{2}$ plants, 409 showed a normal green leaf phenotype and 119 showed the albino phenotype. The segregation ratio of wild-type to albino phenotypes among the $F_{2}$ population was consistent with a 3:1 ratio $\left(\chi^{2}=1.75<\chi^{2} 0.05=3.84\right)$. These results 


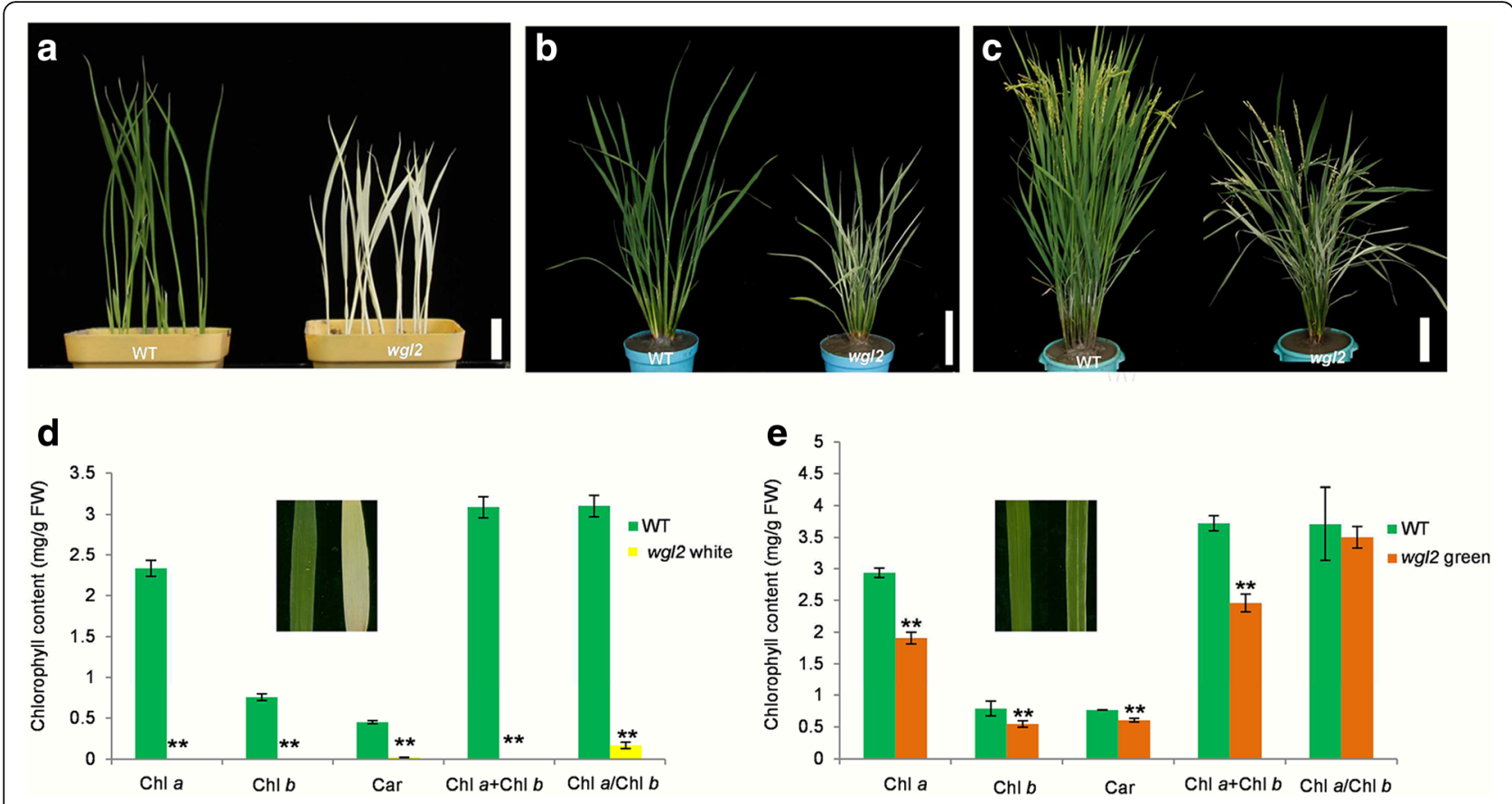

Fig. 1 Phenotypes and pigment contents of wild type and wg/2 plants. a-c Morphology of wild type (WT) and wg/2 plants at the seedling (bar = $2 \mathrm{~cm}$ ), tillering, and heading stages, respectively, in the paddy field $(\mathrm{bar}=10 \mathrm{~cm})$. $\mathbf{d}$ Pigment contents of WT and wgl 2 white seedlings. e Pigment contents of WT and wgl2 green plants that older than the white ones. Chl $a$, chlorophyll $a$; Chl $b$, chlorophyll $b$; Car, carotenoids. Values represent the mean \pm SD of 3 biological replicates. Student's $t$-test was used to generate the $p$ values; ${ }^{* *} p<0.01$

suggest that the wgl2 mutant phenotype is determined by a single recessive nuclear gene.

Using $21 \mathrm{~F}_{2}$ mutant individuals, the WGL2 locus was roughly mapped to the region between the markers A310 and A3-14 on the short arm of chromosome 3
(Fig. 3a). To fine map the WGL2 locus, we designed additional sequence-tagged site markers. By using these and more than 1500 genotypes, the location of WGL2 was narrowed down to a 99-kb region between markers C38 and C3-3, a region that includes 17 putative open

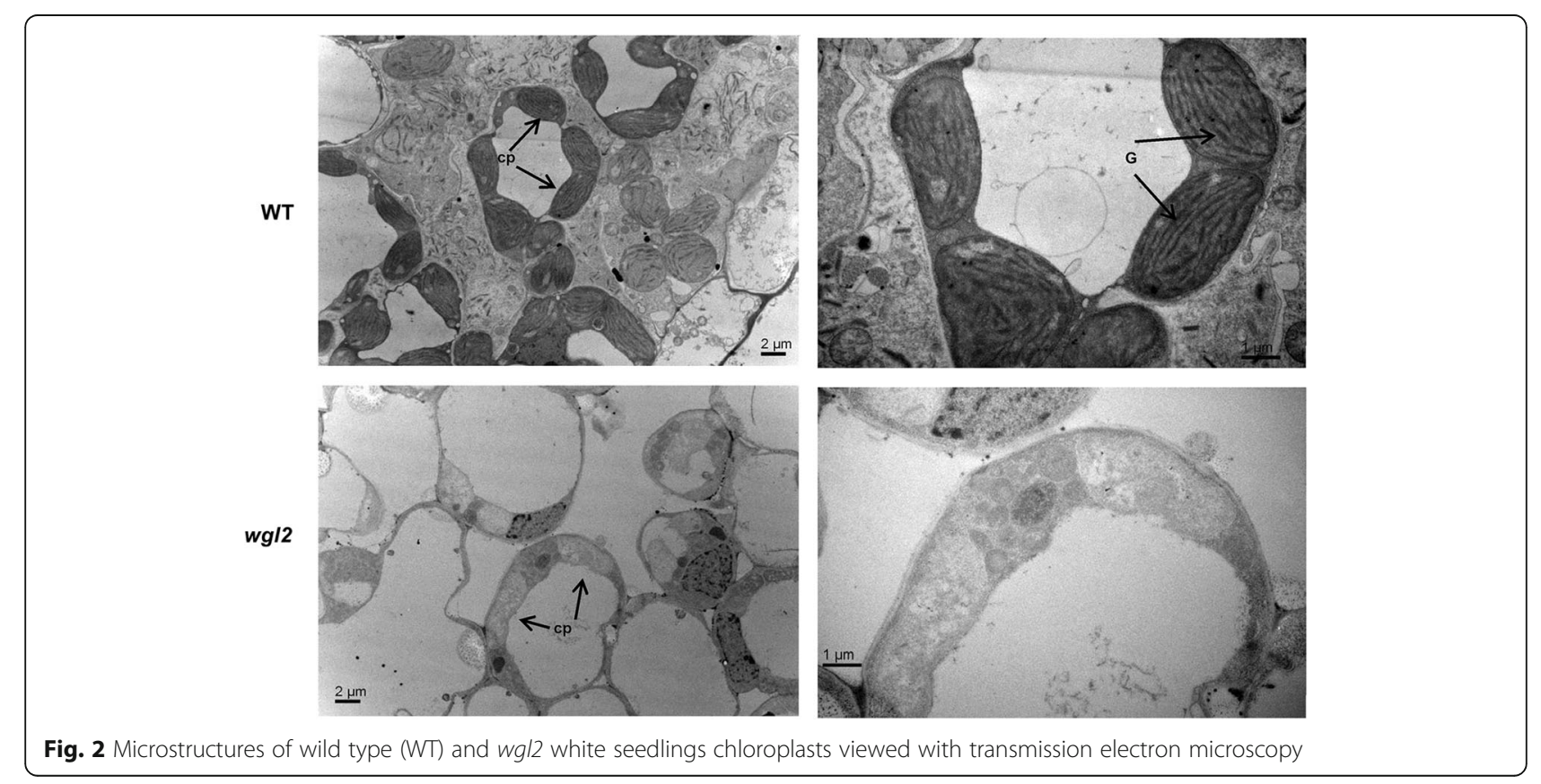




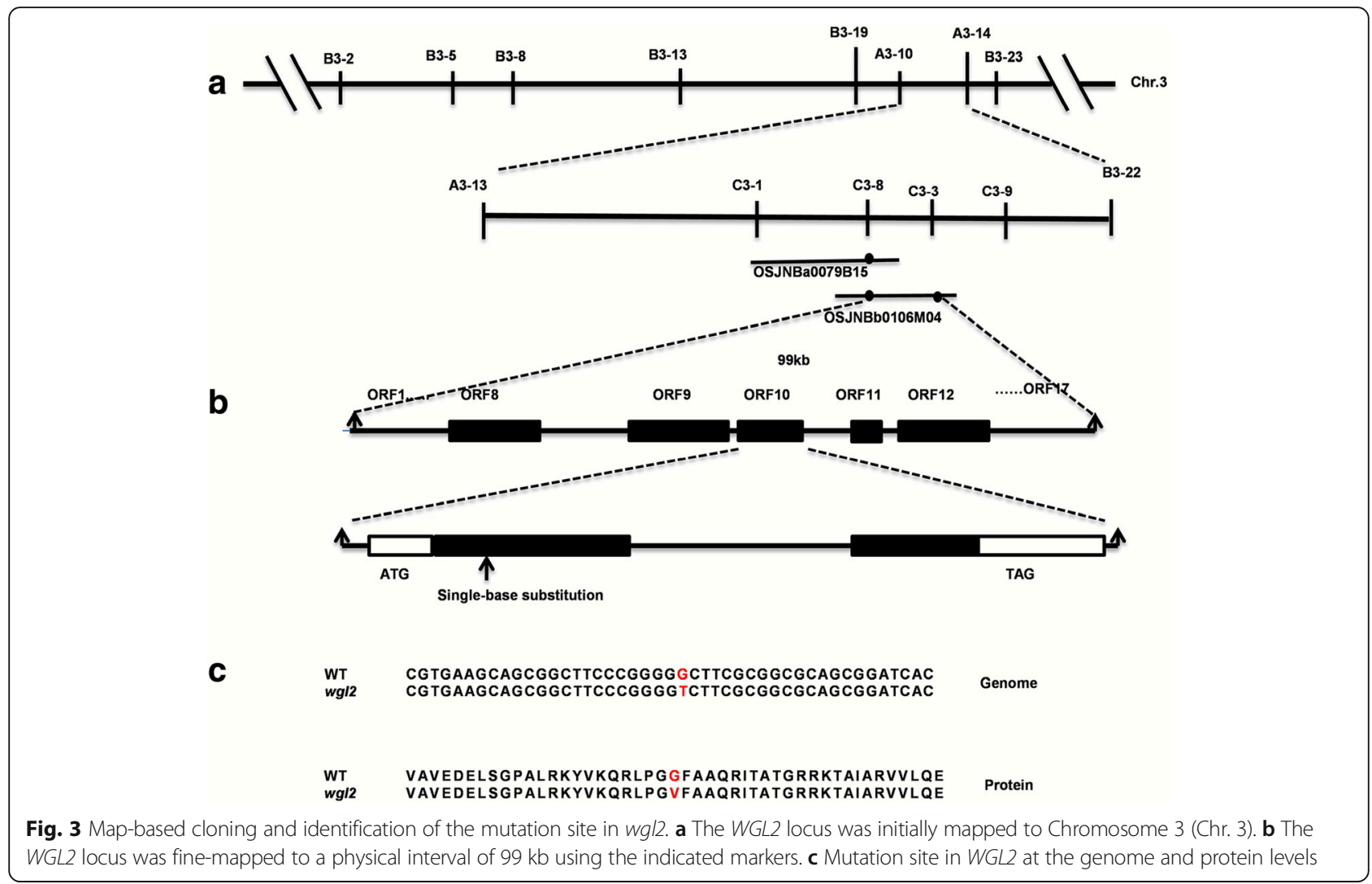

reading frames (ORF: http://rice.plantbiology.msu.edu) (Fig. 3b, Additional file 1: Table S5). DNA sequence analysis of each putative ORF in the wgl2 mutant revealed the presence of a single nucleotide substitution $(\mathrm{G} \rightarrow \mathrm{T})$ in the first exon of LOC_Os03g55930, which resulted in a Gly-to-Val substitution at the 92nd residue (G92 V, Fig. 3c). The gene is 672 bp long, includes two exons and one intron, and encodes a 224 amino acid polypeptide (Fig. 3b). We used Swiss Model (http://swissmodel. expasy.org/) to predict three-dimensional structures for both wild-type and mutant forms of the WGL2 protein and did not observe any obvious structural differences (Biasini et al. 2014).

\section{Complementation and targeted deletion tests}

To confirm that the albino phenotype was attributable to the detected mutation in WGL2, we constructed a complementation vector with a wild-type genomic fragment containing the entire coding region of WGL2, along with 2122 bp of upstream sequence and $281 \mathrm{bp}$ of downstream sequence. The fragment was inserted into the binary vector pCAMBIA1300 to make pCAMBIA1300-WGL2 (Additional file 1: Table S3), which was introduced into the wgl2 mutant by Agrobacterium-mediated transformation. As expected, 15 independent transgenic $\mathrm{T}_{0}$ lines (pCAMBIA1300-WGL2/wgl2) showed a normal phenotype, whereas the wgl2 plants that were transformed with the empty vector showed an albino phenotype that was similar to that of the wgl2 mutant (Fig. 4a). Consistent with this, the $\mathrm{T}_{1}$ plants derived from the $\mathrm{T}_{0}$ plants (pCAMBIA1300-WGL2/wgl2) displayed either green or albino phenotypes, and all of the green seedlings were found to contain the transgene.

Additionally, we used CRISPR/Cas9 to generate mutant alleles of WGL2 in a wild-type background and obtained 9 independent $\mathrm{T}_{0}$ transgenic lines that all carried homozygous mutations. Although each line with a homozygous OsWGL2 phenocopied the wgl2 mutant, it exhibited a seedling lethal phenotype. (Fig. 4c, d). Quantitative RT-PCR analysis demonstrated that WGL2 expression in pCAMBIA1300-WGL2/wgl2 complemented transgenic plants was similar to that in the wild type, whereas WGL2 transcript levels were increased in the $w g l 2$ mutant and in all three independent wgl2cas lines compared to the wild type (Fig. 4b, e). These results support the idea that WGL2 is LOC_Os03g55930 and its mutation is responsible for the $w g l 2$ mutant phenotype.

\section{Subcellular localization of WGL2}

To explore the subcellular localization of WGL2, the full-length WGL2 coding sequence lacking its stop codon was used to construct the pWGL2-GFP vector (Additional file 1: Table S3). The pWGL2-GFP plasmid 


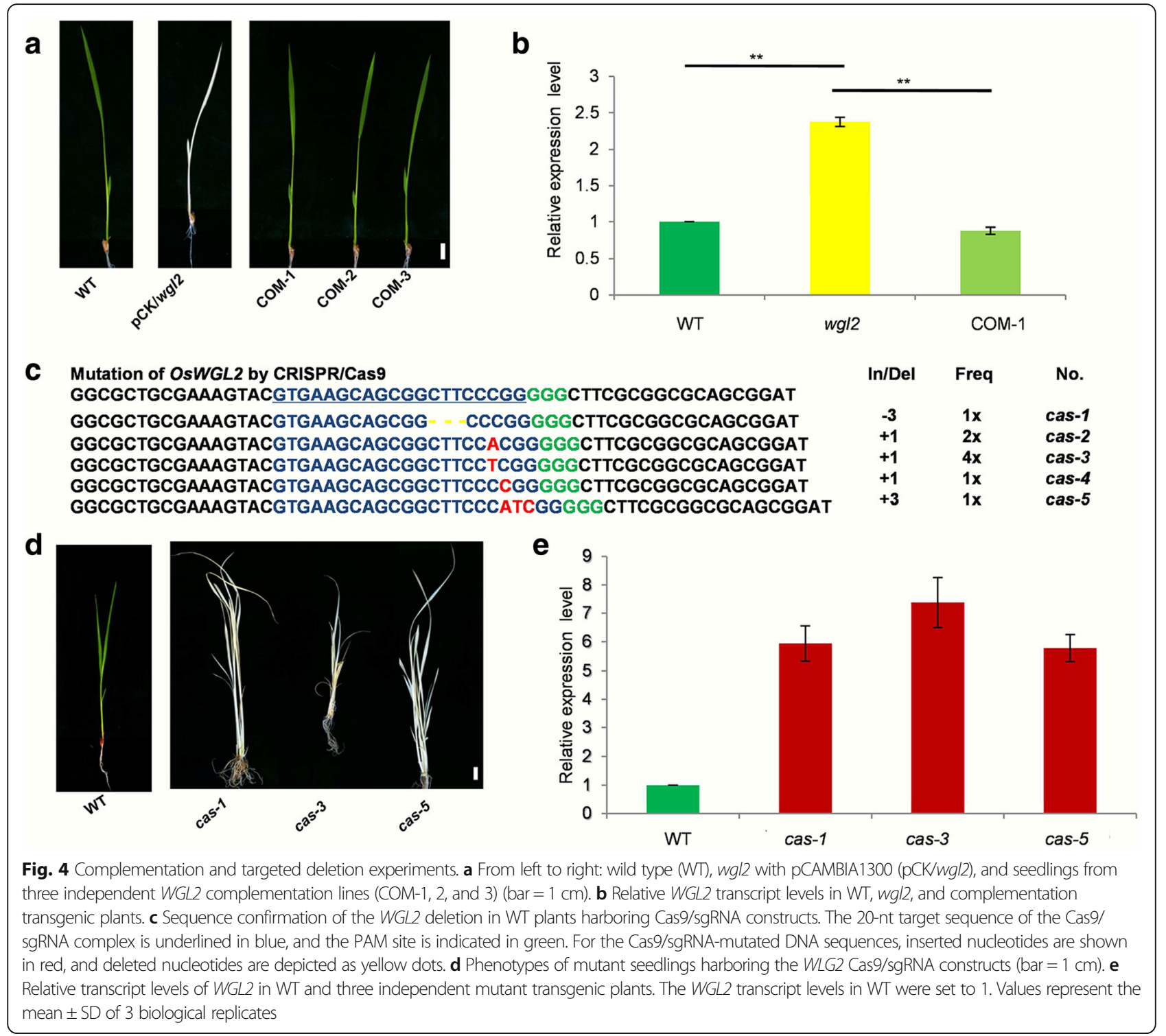

was transformed into rice protoplasts, and an empty GFP vector was used as a negative control. The green fluorescent signal of pWGL2-GFP transformants overlapped with the chlorophyll autofluorescence signal from chloroplasts, whereas the empty GFP vector was observed in the cytoplasm and the nucleus (Fig. 5). These results affirm that WGL2 localizes to the chloroplast.

\section{Phylogenetic analysis of WGL2}

The C-terminal region of chloroplast proteins is often crucial for protein stability, binding with other proteins, photophosphorylation, and trafficking to the chloroplast envelope (Bals et al. 2010; Dünschede et al. 2011; Lung and Chuong 2012; Urbischek et al. 2015). BLAST-P analysis of the NCBI database showed that the WGL2
C-terminal belongs to the ribosomal S9 super family, and is highly conserved in higher plants including Setaria italica, Zea mays, Brachypodium distachyon, Brassica rapa, and Arabidopsis thaliana (Fig. 6a). Among these species, WGL2 from rice exhibited the highest similarity to the orthologs in Sorghum bicolor (81\%), Setaria italic (80\%), and Zea mays (79\%). To investigate the evolutionary relationship among WGL2 homologs, a phylogenic analysis was performed (Fig. 6b). The results show that WGL2 homologues can be clearly divided into two groups: monocots and dicots, and that OsWGL2 is closely related to the grass family containing maize and sorghum. These results demonstrate that the WGL2 protein is a ribosomal S9 protein that is highly conserved in plants. 


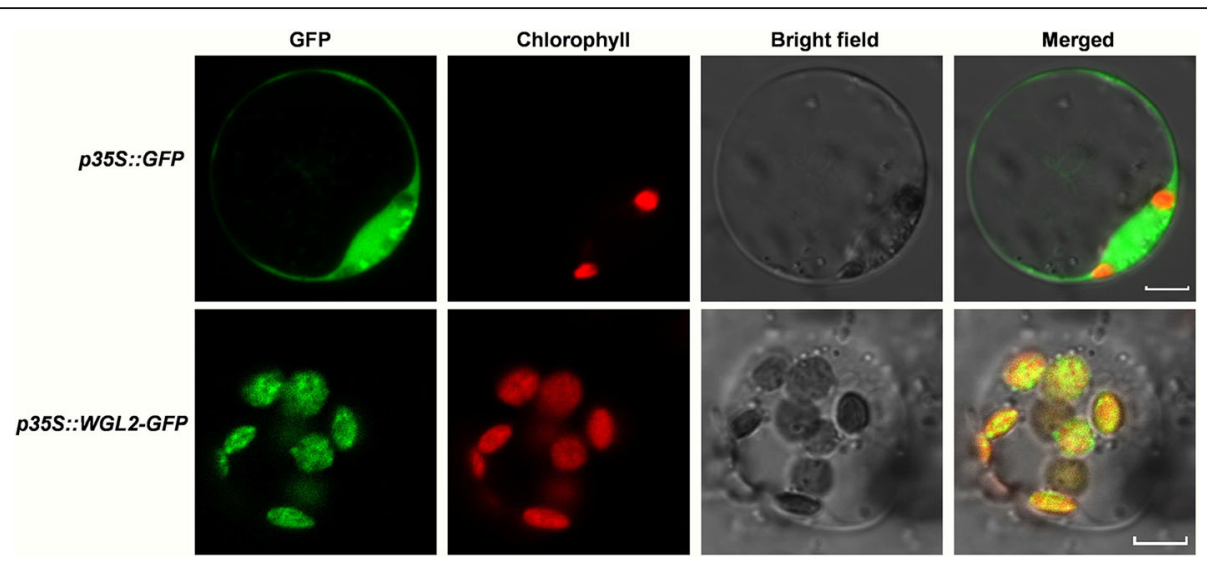

Fig. 5 Subcellular localization of the WGL2-GFP protein in rice protoplasts. GFP signals show that the WGL2-GFP fusion protein produced from the p35S::WGL2-GFP construct localized to the chloroplast and the control GFP localized to the cytoplasm and nucleus. Green fluorescence shows GFP, red fluorescence shows chloroplast autofluorescence, and yellow fluorescence shows the merged fluorescence. (bar $=5 \mu \mathrm{m})$

\section{WGL2 is predominantly expressed in the leaf}

To examine the WGL2 expression pattern in wild-type plants, total RNA was extracted from various organs including young roots, uppermost internodes, flag leaves, leaf sheaths, and young panicles. We detected a dramatic increase in WGL2 expression in flag leaves, and a slight increase in the leaf sheath compared to that in the other organs (Fig. 7). This further supports a role for WGL2 in chloroplast development in rice leaves.

\section{The expression of genes related to chloroplast development, photosynthesis, and chloroplast RNAs is affected in the wgl2 mutant}

Given that WGL2 is a chloroplast nucleoid-localized ribosomal protein, we considered the possibility that it might affect the expression of genes related to chloroplast development. Therefore, we measured transcript levels of 13 representative genes associated with Chl biosynthesis, photosynthesis, and chloroplast development with qRT-PCR (Fig. 8a, c). The transcript levels of the $\mathrm{Chl}$ biosynthesis genes encoding $\mathrm{Mg}$ chelatase $\mathrm{H}$ subunit $(\mathrm{CHLH})$, chlorophyll a oxygenase1 (CAO1), protochlorophyllide A $(P O R A)$, and divinyl reductase $(D V R)$ decreased in the $w g l 2$ albino leaves compared to the wild type (Fig. 8a). However, the transcript level of DVR in $w g l 2$ green leaves that were showed at later stages of development was 2.7 times higher than in wild-type leaves (Fig. 8c).

Chloroplast biogenesis and physiological changes are tightly regulated by the coordination of NEP and PEP. We observed that the expression of PEP-dependent photosynthesis genes including $p s a A$, and $p s b A$, which encode core components of Photosystem I, and $r b c L$, which encodes the large subunit of Rubisco, were lower in $w g l 2$ albino leaves than in the wild-type leaves (Fig. 8a). The expression of NEP-dependent genes, including the RNA polymerase subunit genes RpoB, $R p o C 1$, and $R p o C 2$ was higher in $w g l 2$ albino leaves compared with the wild type. Moreover, the $A t p B$ and AtpE NEP/PEP-dependent genes were strongly inhibited in $w g l 2$ albino leaves compared with the wild type. The expression levels of these genes in $w g l 2$ green leaves were different from the levels in $w g l 2$ albino leaves and in wild-type leaves. For example, the transcript levels of the RNA polymerase subunit genes $\mathrm{RpoB}, \mathrm{RpoC1}$, and $R p o C 2$ were obviously lower in $w g l 2$ green leaves compared with wild-type leaves (Fig. 8c).

In addition, we examined the expression of genes related to ribosome development (Fig. 8b, d). We found that 16S rRNA, 23S rRNA, rps7, and rps18 were severely suppressed in the $w g l 2$ albino mutant compared with the wild type, especially the $16 \mathrm{~S}$ rRNA and $23 \mathrm{~S}$ rRNA that were almost undetectable. Nevertheless, rps10, rps16, rps20, and rpl21 were up-regulated in the wgl2 albino mutant, of which, the increase in rpl21 expression was the most dramatic. When the expression levels of these genes were detected in wgl2 green leaves and wild-type, $16 \mathrm{~S}$ rRNA levels were higher in wgl2 green leaves than in wild-type, and $23 \mathrm{~S}$ rRNA and rps 7 were similar between wgl2 green leaves and wild type; however, the remaining genes were down-regulated in $w g l 2$ green leaves compared with the wild-type. These data show that the mutation in WLG2 results in abnormal expression of genes related to chloroplast development, photosynthesis, and chloroplast RNAs, which may be responsible for the albino phenotype observed in wlg2 albino seedlings.

\section{Discussion}

When chloroplast development and chlorophyll biosynthesis are disrupted, plants usually exhibit a variety of leaf color phenotypes (Jung et al. 2003). Plastid ribosomal proteins are crucial in ribosome biogenesis, 

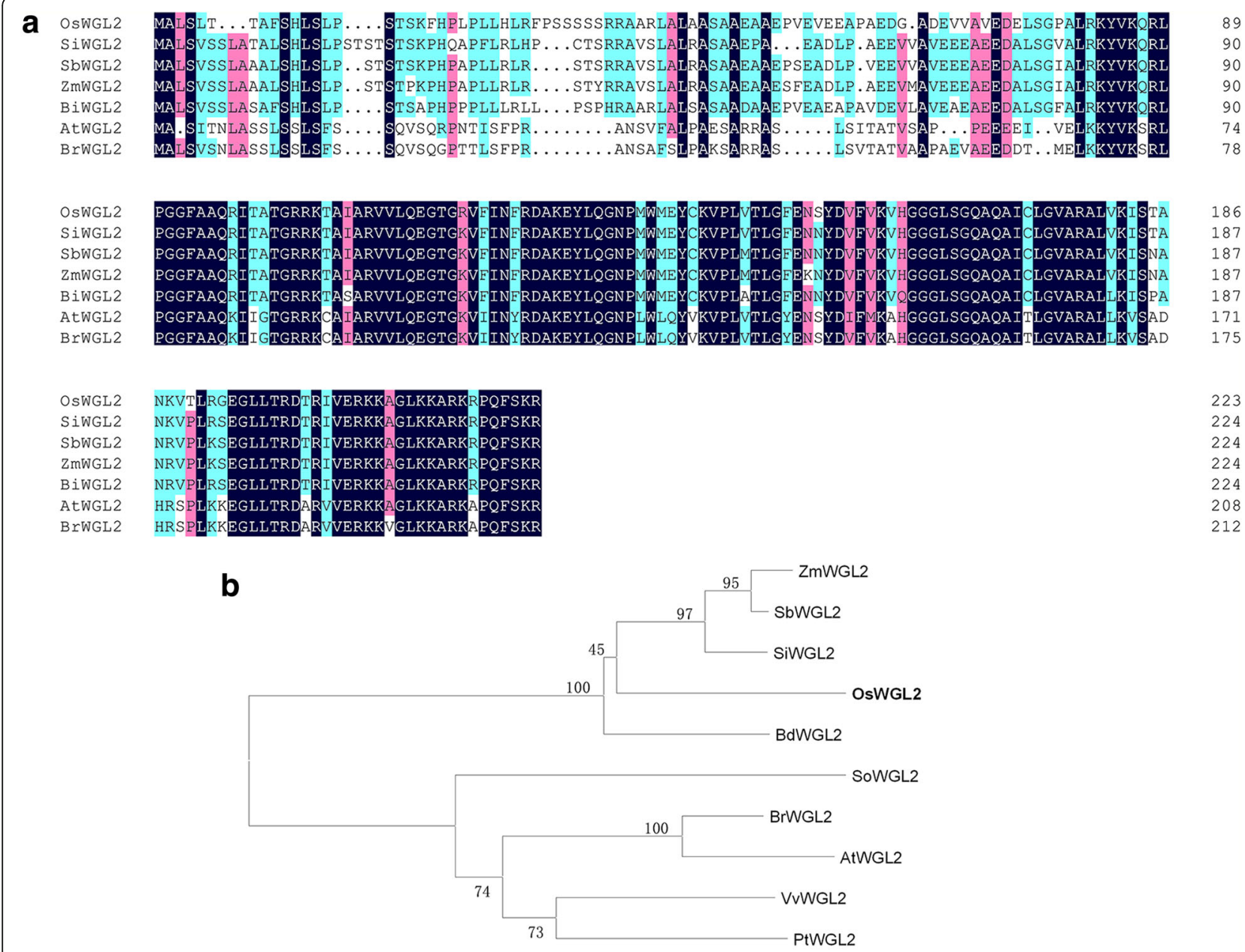

0.050

Fig. 6 Phylogenic analysis of WGL2. a Amino acid sequence alignment of the 7 types of WGL2 homologs. Amino acids that were fully or partially conserved are shaded blue and green, respectively. b Phylogenic tree of OsWGL2 and its homologs. Protein sequences are Setaria italica (SiWGL2, XP_004981623.1), Sorghum bicolor (SbWGL2, XP_002463825.1), Zea mays (ZmWGL2, NP_001 105916.2), Brachypodium distachyon (BdWGL2, XP_003558826.1), Brassica rapa (BrWGL2, XP_009104732.1), Arabidopsis thaliana (AtWGL2, BAD44402.1), Spinacia oleracea (SoWGL2, XP_021845391.1), Vitis vinifera (VVWGL2, XP_002283650.1), Populus trichocarpa (PtWGL2, XP_006375046.1). The tree was constructed using MEGA version 7.0. Scale represents percentage substitutions per site. Statistical support for the nodes is indicated

plastid protein biosynthesis, and early chloroplast development (Lin et al. 2015). Many plastid ribosomal protein mutants have been identified in higher plants, and certain plastid ribosomal proteins have been shown to have roles in diverse biological processes in Arabidopsis (Romani et al. 2012). However, few studies have investigated the functions of PRPs in rice. The first rice PRP mutant reported was the asl1 mutant, which shows an albino lethal phenotype at the seedling stage (Gong et al. 2013). The nuclear gene ASL1 encodes PRPS20 and the asl1 mutation results in abnormal expression of genes related to chlorophyll biosynthesis, photosynthesis, chloroplast development, and plastid ribosome assembly. The rice PRP mutants asl 2 and albino lethal 1 (al1) also show similar albino and seedling lethal phenotypes, as well as a reduction in expression of the 23S rRNA involved in plastid ribosome assembly. ASL2 and AL1 encode PRRL21 and PRPL12, respectively, both of which participate in ribosome assembly (Lin et al. 2015; Zhao et al. 2016). Additionally, the rice PRP mutant white leaf and panicles 1 (wlp1) displays an albino phenotype at low temperatures, which is similar to the thermo-sensitive chlorophyll-deficient mutant 11 (tcd11) mutant. WLP1 encodes PRPL13, and TCD11 encodes PRPS6. Both WLP1 and TCD11 are necessary for normal chloroplast development, especially at low temperatures (Song et al. 2014; Wang et al. 2017a). 


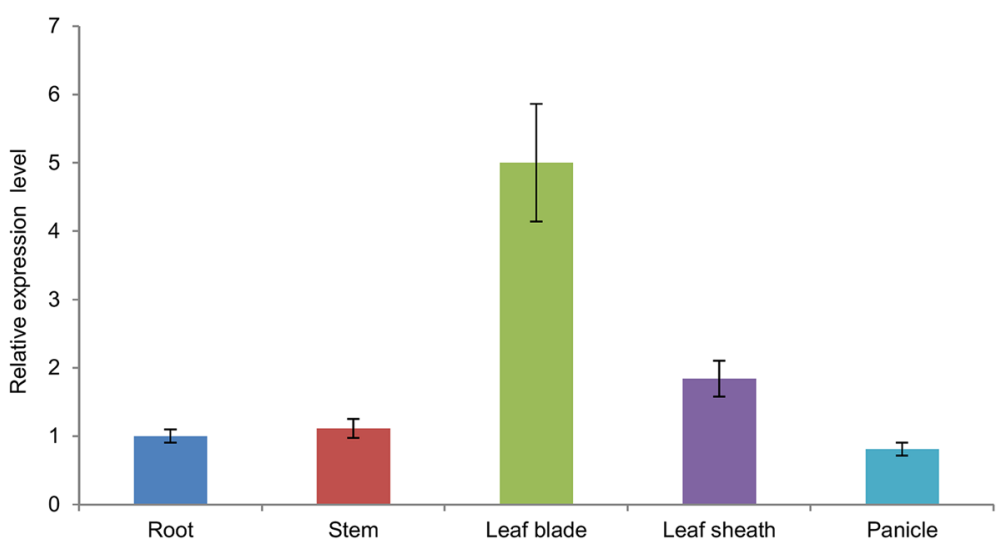

Fig. 7 Relative WGL2 transcript levels in various organs of WT plants. The WGL2 transcript levels in the roots were set to 1. Values represent the mean \pm SD of 3 biological replicates

In this study, a seedling albino rice wgl2 mutant was identified and consistent with its albino phenotype, showed altered pigment content and disordered chloroplast development (Figs. 1a, d and 2). The wgl2 mutant had a single nucleotide substitution $(G \rightarrow T)$ in the first exon of LOC_Os03g55930, which resulted in a
Gly-to-Val substitution at 92nd residue (Fig. 3c). According to the RGAP database (http://rice.plantbiology.msu.edu/index.shtml), WGL2 encodes a ribosomal protein. We found that the WGL2 protein localizes to the chloroplast (Fig. 5). Therefore, we supposed that WGL2 is a type of PRP. Furthermore, a BLASTP analysis in the
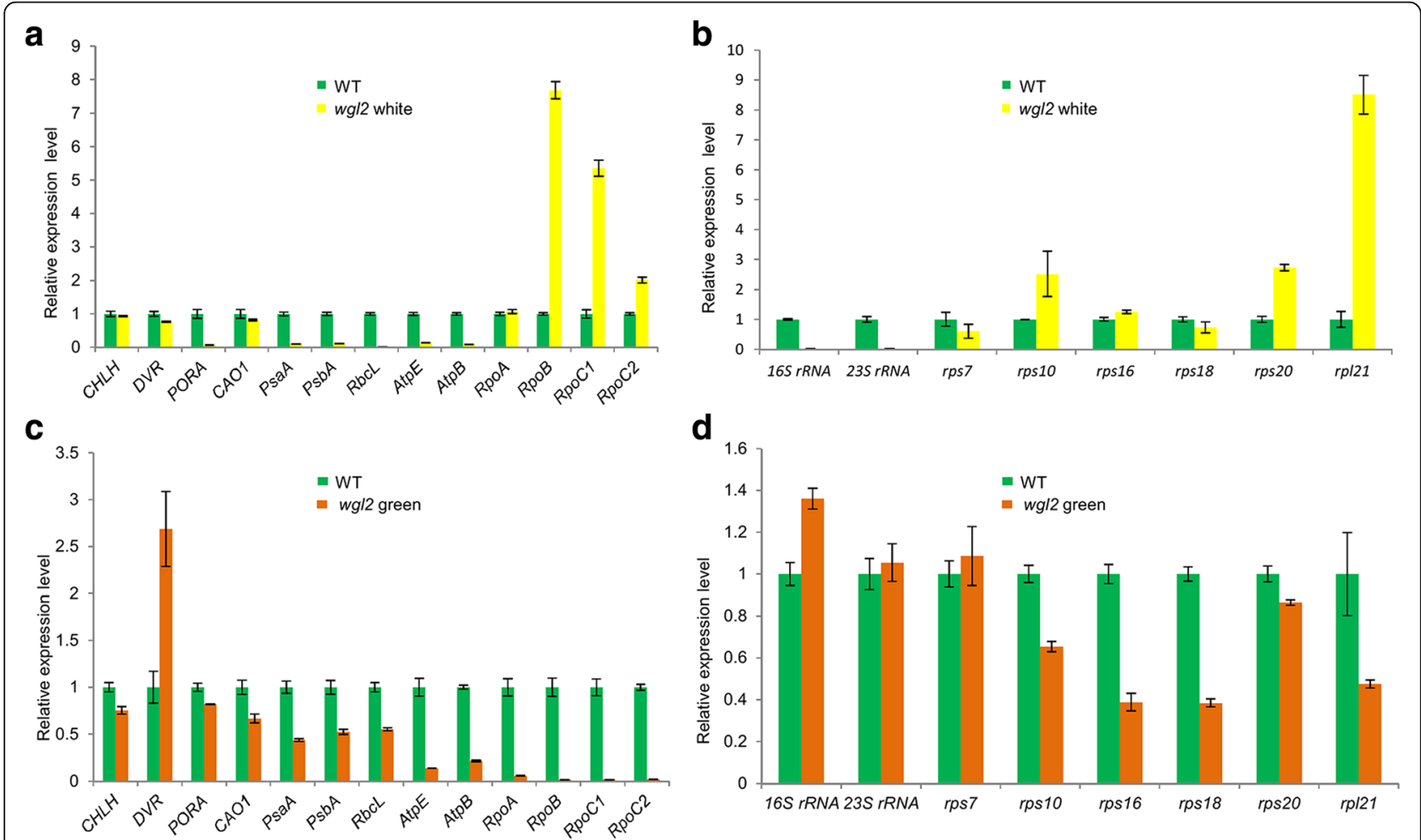

Fig. 8 Expression of genes associated with Chl biosynthesis, photosynthesis, chloroplast development, and ribosome development in the wgl2 mutant and wild type. a, c Expression of genes associated with Chl biosynthesis, photosynthesis, chloroplast development in WT and wg/2 white seedlings (a), and in WT and wgl2 green plants (c). b, d Expression of genes associated with ribosome development in WT and wg/2 white seedlings (b), and in WT and wgl2 green plants (d). The relative expression level of each gene was normalized using UBQ5 as an internal control. The expression level of each gene in WT was set to 1.0 and other samples were calculated accordingly. Values represent the mean \pm SD of 3 biological replicates 
NCBI database showed that the C-terminal of WGL2 belongs to the ribosomal S9 super family and it is highly conserved among chloroplast 30S ribosomal S9 proteins of higher plants (Fig. 6a). According to the NCBI database, WGL2 is the only gene that encodes a ribosomal S9 protein in rice.

The process of plastid formation from a proplastid to a photosynthetically active chloroplast is controlled by NEP and PEP (Hedtke et al. 1997; Shiina et al. 2005). The coordination of NEP and PEP expression plays an important role in chloroplast differentiation. The transcript levels of 13 representative genes associated with Chl biosynthesis, photosynthesis, and chloroplast development were assessed by qRT-PCR (Fig. 8a, c). We found that the expression of PEP-dependent photosynthesis genes, such as $p s a A, p s b A$, and $r b c L$ were severely reduced in the wgl2 mutant, which could possibly be related to the deficiency in chloroplast ribosomes. However, the transcript levels of NEP-dependent genes, such as the RNA polymerase subunits $R p o B, R p o C 1$, and $R p o C 2$ increased in wgl2 albino mutant compared with the wild-type (Fig. 8a), decreased in $w g l 2$ green seedlings compared with the wild-type (Fig. 8c). This increase in expression of NEP genes may be caused through feedback mechanisms to overcome the reduced translation of PEP genes in the chloroplast because NEP preferentially transcribes plastid house-keeping genes, including PEP core subunits and genes associated with basic plastid functions. In addition, the decrease in transcripts of nuclear genes involved in Chl biosynthesis (CHLH, CAO1 and $P O R A$ ) is probably due to the disruption of chloroplast development at the chloroplast build-up step. Consequently, the $w g l 2$ albino or green plants have lower Chl $a$, Chl $b$, and Car contents than the wild type (Fig. 1d, e).

A BLAST-P analysis using the NCBI database shows that WGL2 has high sequence identity with chloroplast $30 \mathrm{~S}$ ribosomal protein $\mathrm{S} 9$ from multiple plant species. Our observations show that the expression of the chloroplast-associated $16 \mathrm{~S}$ and $23 \mathrm{~S}$ rRNAs were dramatically reduced in the $w g l 2$ albino mutant compared to the wild type, and that other genes related to ribosome development also displayed abnormal expression (Fig. 8b). However, most of these genes expression levels in $w g l 2$ green and $w g l 2$ albino were opposite, especially 16 rRNA, rps10, rps20, and rpl21 (Fig. 8b, d). The transcript abundance of WGL2 was higher than the wild type in both the $w g l 2$ point mutant and the CRISPR/ Cas9 transgenic wgl2 mutant lines, which may result from feedback effects (Fig. 4b, e). These results are similar to those observed for the rice wlp1 and al1 mutants (Song et al. 2014; Zhao et al. 2016), and show that mutation of the WGL2 gene hinders Chl biosynthesis, photosynthesis, chloroplast development, and ribosome development in chloroplasts, thereby leading to the observed phenotype in the wgl2 mutant.
RPS9 is widely conserved in yeast, bacteria, protists, mammals, humans, and plants (Wen et al. 2017). RPS9 plays crucial roles in many biological processes such as the early stages of ribosome biogenesis, regulation of mRNA translation, and normal cell growth and proliferation (Ferreiracerca et al. 2007; Lindström and Nistér 2010; Lindström and Zhang 2008; Pnueli and Arava 2007). RPS9 in rice has high sequence identity with maize PRRS9, which is disrupted by the lethal embryo 1 (lem1) mutant; however, the lem1 mutant displays an early embryo lethality phenotype instead of the albino phenotype observed in the wgl2 mutant in rice (Ma and Dooner 2004). In contrast, the null rps9 mutant in bacteria is not lethal, but has a cold-sensitive phenotype. Since single-base substitution is not lethal in the rice $w g l 2$ mutant, the mutant WGL2 protein may be partially functional. Interestingly, we used the CRISPR/Cas9 system to obtain nine independent homozygous $\mathrm{T}_{0}$ transgenic lines, which show albino and seedling-lethal phenotypes, indicating that the OsWGL2 loss-of-function hinders chloroplast development and fails to recover the albino seedling phenotype. These phenotypic differences may be due to different functions of RPS9 in different species and different mutations of RPS9 in the same species.

Many studies have shown that leaf color mutants can transition from abnormal phenotypes to normal phenotypes, and that this transition is primarily affected by environmental conditions, especially light and temperature. The rice temperature-sensitive virescent $(t s v)$ mutant displays a normal green phenotype at high temperatures, whereas the rice heat-stress sensitive albino 1 (hsal) mutant shows normal phenotypes at low temperatures (Qiu et al. 2017; Sun et al. 2017). Light is an important factor in the differentiation of non-photosynthetic proplastids into fully functional, photosynthetic chloroplasts, and affects the expression of genes associated with chloroplast development (Gong et al. 2013; Su et al. 2012; Zhao et al. 1999). The transcript levels of ASL1 and ASL2 were higher when exposed to light than in the dark (Gong et al. 2013; Zhao et al. 2016). In this study, the rice wgl2 mutant exhibited the albino phenotype from germination through the three-leaf stage, and then gradually turned from albino to green during the later stages of development (Fig. 1a, b, c), which may be also affected by environmental conditions. However, further experiments will need to be done to investigate the impact of light and temperature on the $w g l 2$ mutant phenotype to elucidate the role of these environmental factors.

\section{Conclusions}

Rice WGL2 encodes a conserved ribosomal protein, which localizes to the chloroplast. WGL2 is essential for early chloroplast development in rice. These results will facilitate efforts to further uncover the molecular mechanism of chloroplast development. 


\section{Methods}

\section{Plant materials growth conditions}

The wgl2 mutant was obtained by mutagenizing the Oryza sativa japonica cultivar Nipponbare (NPB) with ethyl methane sulfonate (Qian et al. 2016). The $F_{2}$ segregating population was generated by crossing the $w g l 2$ mutant with the indica cultivar Taizhong Native 1 (TN1) and was used for fine mapping wgl2. All plants in this study were grown under natural conditions in the paddy field at the China National Rice Research Institute located in Fuyang, Zhejiang province $\left(119^{\circ} 6^{\prime} \mathrm{E}, 30^{\circ} 0^{\prime} \mathrm{N}\right)$.

\section{Measurement of pigment content}

Pigments were extracted from fresh leaf samples $(0.03 \mathrm{~g})$ from wild-type and $w g l 2$ plants that were cut into small segments and incubated with $3 \mathrm{ml}$ of $80 \%$ acetone in the dark for $48 \mathrm{~h}$. The supernatant was measured with an ultraviolet spectrophotometer (DU800, BECKMAN, Fullerton, USA) at 470, 645, and $663 \mathrm{~nm}$. Total chlorophyll (Chl), Chl $a$, Chl $b$, and carotenoid (Car) contents were measured according to the methods by Arnon (1949) and Wellburn (1994) (Arnon 1949; Wellburn 1994). Three biological replicates were analyzed for each condition.

\section{Transmission electron microscopy (TEM)}

For TEM analysis, transverse sections of leaf samples were taken from the wgl2 white and NPB seedlings grown in the paddy field at the three-leaf stage. Leaf samples were fixed in $2.5 \%$ glutaraldehyde and then in $1 \% \mathrm{OsO}_{4}$. Samples were prepared as described previously (Gothandam et al. 2005), and were observed using a Hitachi H-7650 electron microscope (Tokyo, Japan).

\section{Map-based cloning and vector construction}

To map the WGL2 gene, more than 1500 mutant individuals were selected from the $F_{2}$ population. Genomic DNA (gDNA) was extracted from $F_{2}$ plants with the CTAB method (Jr and Via 1993). Polymorphic markers between NPB and TN1 were identified using simple sequence repeat (SSR) and sequence-tagged site (STS) markers, and were used to map the $w g l 2$ locus. PCR products were separated by agarose gel (4-5\%) electrophoresis. The gDNA fragments containing candidate genes were amplified from mutant and WT and sequenced by the Hangzhou Tsingke Biological Engineering Technology and ServiceCo. Ltd. (Tsingke, Hangzhou, China).

For mutant complementation, the gDNA sequence containing the entire coding region of WGL2, 2122-bp upstream and 281-bp of downstream was amplified and sequenced with the $930 \mathrm{COMF} / \mathrm{R}$ primers (Additional file 1 : Table S3). The resulting fragment was inserted into the binary vector pCAMBIA1300. The pCAMBIA1300-WGL2 vector and control vector (pCAMBIA1300, CK) were introduced into the wgl 2 mutant by Agrobacterium-mediated transformation. For making wgl2 mutants, the target sequence (GTGAAGCAGCGGCTTCCCGG) was chosen using CRISPRdirect (http://crispr.dbcls.jp) and then confirming its specificity (Naito et al. 2015). For construction of the targeted deletion vector, the first round of PCR was performed in two separate reactions with U3tF/tR1tRWGL2 and gR2tF-WGL2/gRNA2R2, and the second round of PCR was performed with U3tF/ gRNA2R2 (Additional file 1: Table S3).The targeted deletion phRubi2-Cas9 vector was constructed for the CRISPR/ Cas9 system (Wang et al. 2017b) and was introduced into NPB by Agrobacterium-mediated transformation.

\section{Phylogenetic analysis}

The predicted full-length WGL2 protein sequence, with 224 amino acids, was obtained from Gramene (http:// www.gramene.org/). The sequences used in the phylogenetic analysis were obtained by a BLASTP search using the WGL2 protein sequence as the query at the National Center for Biotechnology Information (NCBI, http://www.ncbi.nlm.nih.gov/). The full-length amino acid sequences were aligned using the DNAMAN program. A neighbor-joining tree was constructed using MEGA version 7.0 software with the bootstrap method and 1000 replicates. The evolutionary distances were computed using the Poisson correction method and reflect the number of amino acid substitutions. All positions containing gaps and missing data were eliminated.

\section{Subcellular localization}

To investigate the subcellular localization of WGL2, we amplified the full-length WGL2 coding sequence without the termination codon with primers WGL2-GFPF/R (Additional file 1: Table S3). The resulting fragment was introduced into the GFP vector pCA1301-35S-S65 T-GFP (Ren et al. 2017). The WGL2-GFP vector was transformed into rice protoplasts (Yu et al. 2014), and the transformed protoplasts were observed with a Zeiss LSM700 laser scanning confocal microscope (Car Zeiss, Inc., Thornwood, NY, USA).

\section{RNA extraction and qRT-PCR analysis}

Total RNA was extracted and purified from different tissues using the AxyPrep total RNA Miniprep Kit (Axygen) according to the manufacturer's instructions. The cDNA was reverse transcribed using the ReverTraAce quantitative PCR RT Master Mix Kit with gDNA remover (Toyobo) according to the manufacturer's instructions. Quantitative reverse transcription PCR (qRT-PCR) was performed with a CFX96 Touch Real-time PCR Detection System using the $2 \times$ SsoFast EvaGreen SuperMix (Bio-Rad). The rice $U B Q 5$ gene was used as an internal control. All primers for qRT-PCR are listed in Additional file 1: 
Table S2. The data were expressed as the mean \pm SD of three biological replicates. A Student's $t$-test was used for statistical analysis.

\section{Additional file}

Additional file 1: Table S1. Markers used for fine mapping and sequencing of the WGL2 locus. Table S2. RT-PCR primers. Table S3. Vector construction primers. Table S4. Effect of mutations in cas on WGL2. Table S5. Predicted genes in the mapped region. (DOCX $31 \mathrm{~kb}$ )

\section{Funding}

The work was supported by the National Natural Science Foundation of China (Grant No. 31601284); The Transgenic Plant Research and Commercialization Project of the Ministry of Agriculture of China (Grant No. 2016ZX08001003-002); Zhejiang Province Outstanding Youth Fund (Grant No. LR16C130001); The Collaborative Innovation Project of the Chinese Academy of Agricultural Sciences; and the State Key Laboratory of Rice Biology Research Project (2017ZZKT10103).

\section{Availability of data and materials}

All relevant data are provided as figures within the paper.

\section{Authors' contributions}

$L Z, Z Q$, and DC designed the research, performed the experiments, analyzed the data, and wrote the manuscript. LH, SZ, ZY, YZ, ZW, DR, and QQ performed the experiments and analyzed the data. LG provided a critical assessment of the manuscript. ZQ, LZ and LG conceived the project, designed the research, analyzed the data, and wrote the manuscript. All authors read and approved the final manuscript.

\section{Ethics approval and consent to participate}

There are no ethics issues associated with this article.

\section{Consent for publication}

These co-authors involved in the paper all consent to publish this article in Rice.

\section{Competing interests}

The authors declare that they have no competing interests.

\section{Publisher's Note}

Springer Nature remains neutral with regard to jurisdictional claims in published maps and institutional affiliations.

\section{Author details}

'State Key Laboratory of Rice Biology, China National Rice Research Institute, Hangzhou 310006, China. ${ }^{2}$ Biotechnology Research Center, Chongqing Academy of Agricultural Sciences, Chongqing 401329, China.

Received: 14 March 2018 Accepted: 4 July 2018

Published online: 11 July 2018

\section{References}

Arnon DI (1949) Copper enzymes in isolated chloroplasts. Polyphenoloxidase in Beta vulgaris. Plant Physiol 24:1

Bals T, Dunschede B, Funke S, Schunemann D (2010) Interplay between the cpSRP pathway components, the substrate LHCP and the translocase Alb3: an in vivo and in vitro study. FEBS Lett 584:4138-4144

Biasini M, Bienert S, Waterhouse A, Arnold K, Studer G, Schmidt T, Kiefer F, Gallo Cassarino T, Bertoni M, Bordoli L, Schwede T (2014) SWISS-MODEL: modelling protein tertiary and quaternary structure using evolutionary information. Nucleic Acids Res 42:W252-W258

De Santis-Maclossek G, Kofer W, Bock A, Schoch S, Maier RM, Wanner G, Rudiger W, Koop HU, Herrmann RG (1999) Targeted disruption of the plastid RNA polymerase genes rpoA, B and C1: molecular biology, biochemistry and ultrastructure. Plant J 18:477-489
Dünschede B, Bals T, Funke S, Schünemann D (2011) Interaction studies between the chloroplast signal recognition particle subunit cpSRP43 and the fulllength translocase Alb3 reveal a membrane-embedded binding region in Alb3 protein. J Biol Chem 286:35187-35195

Ehrnthaler M, Scharff LB, Fleischmann TT, Hasse C, Ruf S, Bock R (2014) Synthetic lethality in the tobacco plastid ribosome and its rescue at elevated growth temperatures. Plant Cell 26:765-776

Ferreiracerca S, Pöll G, Kühn H, Neueder A, Jakob S, Tschochner H, Milkereit P (2007) Analysis of the in vivo assembly pathway of eukaryotic 405 ribosomal proteins. Mol Cell 28:446

Gong X, Jiang Q, Xu J, Zhang J, Teng S, Lin D, Dong Y (2013) Disruption of the rice plastid ribosomal protein $s 20$ leads to chloroplast developmental defects and seedling lethality. G3 (Bethesda) 3:1769-1777

Gothandam KM, Kim ES, Cho H, Chung YY (2005) OsPPR1, a pentatricopeptide repeat protein of rice is essential for the chloroplast biogenesis. Plant Mol Biol 58:421-433

Hajdukiewicz PTJ, Allison LA, Maliga P (1997) The two RNA polymerases encoded by the nuclear and the plastid compartments transcribe distinct groups of genes in tobacco plastids. EMBO J 16:4041-4048

He L, Zhang S, Qiu Z, Zhao J, Nie W, Lin H, Zhu Z, Zeng D, Qian Q, Zhu L (2018) FRUCTOKINASE-LIKE PROTEIN 1 interacts with TRXZ to regulate chloroplast development in rice. J Integr Plant Biol

Hedtke B, Börner T, Weihe A (1997) Mitochondrial and chloroplast phage-type RNA polymerases in Arabidopsis. Science 277:809

Jr SC, Via LE (1993) A rapid CTAB DNA isolation technique useful for RAPD fingerprinting and other PCR applications. Biotechniques 14:748-750

Jung KH, Hur J, Ryu CH, Choi Y, Chung YY, Miyao A, Hirochika H, An G (2003) Characterization of a rice chlorophyll-deficient mutant using the T-DNA gene-trap system. Plant Cell Physiol 44:463-472

Kusumi K, Chono Y, Shimada H, Gotoh E, Tsuyama M, Iba K (2010) Chloroplast biogenesis during the early stage of leaf development in rice. Plant Biotechnol 27:85-90

Kusumi K, Sakata C, Nakamura T, Kawasaki S, Yoshimura A, Iba K (2011) A plastid protein NUS1 is essential for build-up of the genetic system for early chloroplast development under cold stress conditions. Plant J 68:1039-1050

Lin D, Jiang Q, Zheng K, Chen S, Zhou H, Gong X, Xu J, Teng S, Dong Y (2015) Mutation of the rice ASL2 gene encoding plastid ribosomal protein $L 21$ causes chloroplast developmental defects and seedling death. Plant Biol (Stuttg) 17:599-607

Lindström MS, Nistér M (2010) Silencing of ribosomal protein S9 elicits a multitude of cellular responses inhibiting the growth of cancer cells subsequent to p53 activation. PLoS One 5:e9578

Lindström MS, Zhang Y (2008) Ribosomal protein S9 is a novel B23/NPM-binding protein required for normal cell proliferation. J Biol Chem 283:15568

Lung SC, Chuong SD (2012) A transit peptide-like sorting signal at the C terminus directs the Bienertia sinuspersici preprotein receptor Toc159 to the chloroplast outer membrane. Plant Cell 24:1560-1578

Ma Z, Dooner HK (2004) A mutation in the nuclear-encoded plastid ribosomal protein $S 9$ leads to early embryo lethality in maize. Plant J 37:92-103

Mullet JE (1993) Dynamic regulation of chloroplast transcription. Plant Physiol 103:309-313

Naito Y, Hino K, Bono H, Ui-Tei K (2015) CRISPRdirect: software for designing CRISPR/Cas guide RNA with reduced off-target sites. Bioinformatics (Oxford England) 31:1120-1123

Pnueli L, Arava Y (2007) Genome-wide polysomal analysis of a yeast strain with mutated ribosomal protein S9. BMC Genomics 8:285

Pogson BJ, Albrecht V (2011) Genetic dissection of chloroplast biogenesis and development: an overview. Plant Physiol 155:1545-1551

Qian Q, Guo L, Smith SM, Li J (2016) Breeding high-yield superior quality hybrid super rice by rational design. Nat Sci Rev 3:283-294

Qiu Z, Kang S, He L, Zhao J, Zhang S, Hu J, Zeng D, Zhang G, Dong G, Gao Z, Ren D, Chen G, Guo L, Qian Q, Zhu L (2017) The newly identified heat-stress sensitive albino 1 gene affects chloroplast development in rice. Plant Sci

Ren D, Yu H, Rao Y, Xu Q, Zhou T, Hu J, Zhang Y, Zhang G, Zhu L, Gao Z (2017) "Two-florets spikelet" as a novel resource has the potential to increase rice yield. Plant Biotechnol J

Rogalski M, Ruf S, Bock R (2006) Tobacco plastid ribosomal protein S18 is essential for cell survival. Nucleic Acids Res 34:4537-4545

Rogalski M, Schöttler MA, Thiele W, Schulze WX, Bock R (2008) Rpl33, a nonessential plastid-encoded ribosomal protein in tobacco, is required under cold stress conditions. Plant Cell 20:2221-2237 
Romani I, Tadini L, Rossi F, Masiero S, Pribil M, Jahns P, Kater M, Leister D, Pesaresi P (2012) Versatile roles of Arabidopsis plastid ribosomal proteins in plant growth and development. Plant J 72:922-934

Sakamoto W, Uno Y, Quan Z, Miura E, Kato Y, Sodmergen, Hayashi M, Nishimura M (2009) Arrested differentiation of proplastids into chloroplasts in variegated leaves characterized by plastid ultrastructure and nucleoid morphology. Plant Cell Physiol 50:2069-2083

Schultes NP, Sawers RJH, Brutnell TP, Krueger RW (2000) Maize high chlorophyll fluorescent 60 mutation is caused by an ac disruption of the gene encoding the chloroplast ribosomal small subunit protein 17. Plant J 21:317-327

Shiina T, Tsunoyama Y, Nakahira Y, Khan MS (2005) Plastid RNA polymerases, promoters, and transcription regulators in higher plants. Int Rev Cytol 244:1

Song J, Wei X, Shao G, Sheng Z, Chen D, Liu C, Jiao G, Xie L, Tang S, Hu P (2014) The rice nuclear gene WLP1 encoding a chloroplast ribosome $L 13$ protein is needed for chloroplast development in rice grown under low temperature conditions. Plant Mol Biol 84:301-314

Su N, Hu ML, Wu DX, Wu FQ, Fei GL, Lan Y, Chen XL, Shu XL, Zhang X, Guo XP (2012) Disruption of a Rice Pentatricopeptide repeat protein causes a seedling-specific albino phenotype and its utilization to enhance seed purity in hybrid Rice production. Plant Physiol 159:227-238

Subramanian AR (1985) The ribosome: its evolutionary diversity and the functional role of one of its components. Essays Biochem 21:45-85

Sun J, Zheng T, Yu J, Wu T, Wang X, Chen G, Tian Y, Zhang H, Wang Y, Terzaghi W (2017) TSV, a putative plastidic oxidoreductase, protects rice chloroplasts from cold stress during development by interacting with plastidic thioredoxin Z. New Phytol 215:240-255

Tiller N, Bock R (2014) The translational apparatus of plastids and its role in plant development. Mol Plant 7:1105-1120

Urbischek M, Braun SNV, Brylok T, Gügel IL, Richter A, Koskela M, Grimm B, Mulo P, Bölter B, Soll J (2015) The extreme Albino3 (Alb3) C terminus is required for Alb3 stability and function in Arabidopsis thaliana. Planta 242:733-746

Wang WJ, Zheng KL, Gong XD, Xu JL, Huang JR, Lin DZ, Dong YJ (2017a) The rice TCD11 encoding plastid ribosomal protein $\mathrm{S} 6$ is essential for chloroplast development at low temperature. Plant Sci 259:1-11

Wang ZW, Lv J, Xie SZ, Zhang Y, Qiu ZN, Chen P, Cui YT, Niu YF, Hu SK, Jiang HZ (2017b) OsSLA4 encodes a pentatricopeptide repeat protein essential for early chloroplast development and seedling growth in rice. Plant Growth Regul:1-12

Wellburn AR (1994) The spectral determination of chlorophylls a and b, as well as Total carotenoids, using various solvents with spectrophotometers of different resolution. J Plant Physiol 144:307-313

Wen L, Lü G, Lu S, Gao H, Chen B, Ma Y, Yuan Y, Zhang H, Xiao Y, Liu H (2017) Molecular cloning and characterization of ribosomal protein $S 9$ in echinococcus granulosus. J Parasitol 103(6):699-707

Yamaguchi K, Beligni MV, Prieto S, Haynes PA, Mcdonald WH, Mayfield SP (2003) Proteomic characterization of the Chlamydomonas reinhardtii chloroplast ribosome. Identification of proteins unique to th e70 S ribosome. J Biol Chem 278:33774-33785

Yamaguchi K, Subramanian AR (2003) Proteomic identification of all plastidspecific ribosomal proteins in higher plant chloroplast 305 ribosomal subunit - PSRP-2 (U1A-type domains), PSRP-3 alpha/beta (ycf65 homologue) and PSRP-4 (thx homologue). Eur J Biochem 270:190-205

Yu C, Wang L, Chen C, He C, Hu J, Zhu Y, Huang W (2014) Protoplast: a more efficient system to study nucleo-cytoplasmic interactions. Biochem Biophys Res Commun 450:1575-1580

Zhang J, Yuan H, Yang Y, Fish T, Lyi SM, Thannhauser TW, Zhang L, Li L (2016) Plastid ribosomal protein S5 is involved in photosynthesis, plant development, and cold stress tolerance in Arabidopsis. J Exp Bot 67: $2731-2744$

Zhao DS, Zhang CQ, Li QF, Yang QQ, Gu MH, Liu QQ (2016) A residue substitution in the plastid ribosomal protein L12/AL1 produces defective plastid ribosome and causes early seedling lethality in rice. Plant Mol Biol 91:161-177

Zhao YY, Xu T, Zucchi P, Bogorad L (1999) Subpopulations of chloroplast ribosomes change during photoregulated development of Zea mays leaves: ribosomal proteins L2, L21, and L29. Proc Natl Acad Sci U S A 96:8997-9002

\section{Submit your manuscript to a SpringerOpen ${ }^{\circ}$ journal and benefit from:}

- Convenient online submission

- Rigorous peer review

- Open access: articles freely available online

- High visibility within the field

- Retaining the copyright to your article

Submit your next manuscript at $\boldsymbol{\nabla}$ springeropen.com 